\title{
Tarefa experimental de metamemória para avaliar monitoramento e controle de memória
}

\author{
Maxciel Zortea - Universidade Federal do Rio Grande do Sul, Porto Alegre, Brasil \\ Graciela Inchausti de Jou - Universidade Federal do Rio Grande do Sul, Porto Alegre, Brasil \\ Jerusa Fumagalli de Salles - Universidade Federal do Rio Grande do Sul, Porto Alegre, Brasil
}

\section{Resumo}

Tarefas experimentais para avaliar processos de monitoramento e controle de memória permitem o exame online dessas habilidades. No âmbito nacional, estas tarefas ainda precisam ser desenvolvidas, facilitando e incentivando projetos de pesquisa futuros. Este artigo apresenta os passos para a construção de uma tarefa para avaliação dos processos de monitoramento e controle. No Estudo 1, é descrita a seleção de 100 estímulos (pares de palavra pista-alvo) e suas características psicolinguísticas. O Estudo 2 apresenta dados do estudo piloto da tarefa com dez adultos, com foco na avaliação do monitoramento. No Estudo 3, são apresentados dados de processos de monitoramento e controle num estudo piloto com 12 universitários, um caso clínico pós-AVC e dois idosos neurologicamente saudáveis. Os resultados apontaram a necessidade de duas versões da tarefa, para diferentes populações, que permitisse a variabilidade dos dados intra e entre participantes. Também surgiram achados preliminares pertinentes a importantes hipóteses da área da metamemória.

Palavras-chave: Metacognição; Memória; Monitoramento; Controle; Delineamento experimental.

\section{Metamemory experimental task to evaluate memory monitoring and control}

\begin{abstract}
Experimental tasks for the assessment of monitoring and control memory processes allow the online exam of these abilities. In the national field these tasks still need to be elaborated in order to facilitate and stimulate future research projects. This article presents the steps for the elaboration of a task for examining memory monitoring and control processes. In Study 1 we describe the selection of 100 stimuli (cue-target word pairs) and its psycholinguistic aspects. Study 2 presents data of a pilot-study of the task with ten adults, focusing on the evaluation of monitoring processes. In Study 3 we show data of monitoring and control processes of a pilot-study with 12 undergraduate students as well as a clinical case of stroke and two neurologically unimpaired elderly people. Results suggest the need of two different versions of the task for different populations, which would allow enough variance within and between participants. In addition, other preliminary findings relevant for current metamemory hypothesis are presented.

Keywords: Metacognition; Memory; Monitoring; Control; Experimental design.
\end{abstract}

Tarea experimental de metamemoria para evaluar monitoreo y control de memoria

\section{Resumen}

Tareas experimentales para evaluar procesos de monitoreo y control de la memoria permiten el examen online de estas habilidades. A nivel nacional, estas tareas todavía necesitan ser desarrolladas, para facilitar y promover futuros proyectos de investigación. Este estudio presenta los pasos ejecutados para la construcción de una tarea para evaluar los procesos de monitoreo y control. El Estudio 1 describe la selección de 100 pares de estímulos (señal-test) y sus características psicolingüísticas. El Estudio 2 presenta datos de un estudio piloto con diez adultos, centrándose en la evaluación del monitoreo. En el Estudio 3 se presentan los datos de procesos de monitoreo y control de un estudio piloto con 12 universitarios, un caso clínico de accidente cerebrovascular y dos ancianos neurológicamente sanos. Los resultados indicaron la necesidad de dos versiones de la tarea, para diferentes poblaciones, permitiendo variabilidad de los datos dentro y entre los participantes. Además, surgieron otros hallazgos preliminares pertinentes a importantes hipótesis en metamemoria.

Palabras-clave: Metacognición; Memoria; Monitoreo; Control; Delineamiento experimental.

\section{Introdução}

Este trabalho tem por objetivo apresentar os passos do desenvolvimento de uma tarefa experimental para investigação dos processos de monitoramento e controle de memória. Paradigmas experimentais computadorizados para o estudo desses processos de metamemória são comumente utilizados no âmbito internacional (Dunlosky \& Nelson, 1994; Metcalfe \& Kornell, 2003). Contudo, no Brasil não foi encontrada, até o presente momento, uma tarefa que permita a avaliação online de alguns processos de metamemória e que apresente estímulos verbais em português brasileiro, com características psicolinguísticas controladas e que disponha de estudos-piloto para adequação dos procedimentos. Desse modo, pesquisadores e profissionais das áreas da Psicologia Experimental, Neuropsicologia Cognitiva, Educação, Neuroimagem, Neurociência Computacional, Avaliação e Reabilitação Neuropsicológicas e demais áreas das Neurociências Cognitivas que pesquisam com seres humanos podem se beneficiar do uso desta tarefa. 


\section{Modelos teóricos e medidas de metamemória}

Em 1977, reunindo trabalhos de outros autores, John H. Flavell inaugurou a área de estudos da metamemória (Veenman, Van Hout-Wolters \& Afflerbach, 2006), a qual faria parte, em 1979, dos estudos em metacognição (Flavell, 1979). A metamemória consiste na capacidade ou habilidade de uma pessoa em pensar sobre sua própria memória (Koriat, 1993). Segundo o modelo de processamento de informação bastante consolidado de Nelson e Narens (1990), os processos de memória, tais como codificação, armazenamento e recuperação, são acompanhados simultaneamente por processos metamnemônicos. Portanto, o modelo cognitivo assume dois níveis de funcionamento, um nivel-meta e um nivel-objeto, conforme a Figura 1. Nelson e Narens propõem que a capacidade metamnemônica envolve dois processos: monitoramento e controle. $\mathrm{O}$ monitoramento refere-se à habilidade do nível-meta ter acesso às mudanças que ocorrem no nível-objeto e o controle refere-se à modificação, por parte do nível-meta, das operações que se dão no nível-objeto (Nelson \& Narens, 1990). Isso significa que, por meio do monitoramento, o indivíduo pode observar e realizar julgamentos sobre os próprios processos de memória, e, por meio do controle, o indivíduo pode regular os processos de memória em função do que foi ou está sendo monitorado. Ao julgar como está memorizando ou aprendendo determinada informação, o indivíduo pode alocar diferentes estratégias de aprendizagem.

Portanto, é através da precisão dos julgamentos específicos realizados ao longo dos processos de codificação, armazenamento e recuperação de memória que se estuda a capacidade de monitoramento e é através das estratégias de estudo que se investiga a capacidade de controle dos processos de memória.

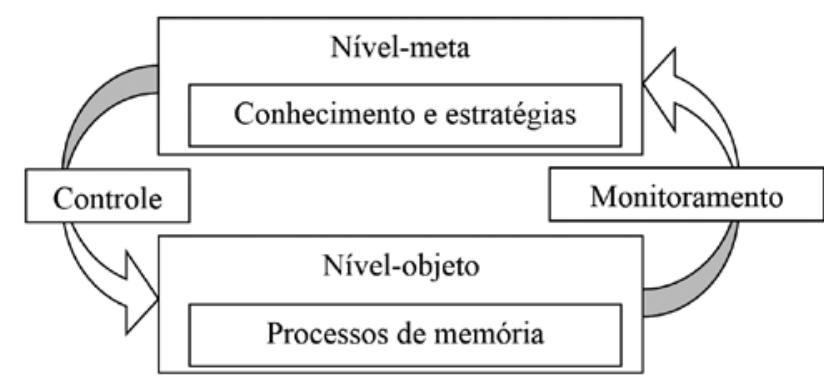

Figura 1. Modelo de Nelson e Narens (1990), resumido por Van Overshelde (2008), adaptado pelos autores, demonstrando a relação entre monitoramento, controle e conhecimento.
Entre os julgamentos metacognitivos utilizados para pesquisar o monitoramento, estão os julgamentos de aprendizagem (judgments of learning ou JOL) e os julgamentos de sensação de saber (feeling-of-knowing ou FOK judgments). Os primeiros são realizados imediatamente ou após alguns minutos (JOLs tardios) da codificação de um item e têm como base o quanto o indivíduo julga que conseguirá lembrar desse item posteriormente. $\mathrm{O}$ segundo tipo de julgamento é feito para itens que não puderam ser recuperados, de modo que o indivíduo refere o quanto julga que conseguirá reconhecer corretamente o item quando apresentado entre distratores (Leonesio \& Nelson, 1990). Ou seja, apesar de não conseguir lembrar qual era o item, o participante relata o quanto "sente que sabe" a resposta certa. Para avaliação desses julgamentos, em uma tarefa, são criados itens do tipo pista-alvo na fase de estudo. O par pista-alvo pode ser, por exemplo, uma pergunta e uma resposta de conhecimento geral (Marquié \& Huet, 2000), a face e o nome de uma pessoa (Chua, Schacter \& Sperling, 2009), ou um par de palavras (Koriat, Ma'ayan \& Nussinson, 2006; Thomas, Bulevich \& Dubois, 2012), conforme será usado no presente estudo. No caso de pares de palavras, no Brasil há normas de associação de palavras para adultos (Salles e cols., 2008). Durante os julgamentos, pode ser apresentada apenas a pista, para o participante identificar o item a ser julgado.

Existem alguns modelos teóricos que buscam explicar como esses julgamentos metamnemônicos ocorrem. Atualmente a abordagem da utilização de pistas (Koriat, 1997; Koriat \& Levy-Sadot, 2001) é a mais aceita, e sustenta que os julgamentos são inferenciais e têm como base uma série de pistas que, quando válidas, resultam em uma boa predição do desempenho de memória. Essas pistas não são as mesmas das pistas enquanto estímulos (pista-alvo), apesar de o termo ser o mesmo (cue). Essas pistas podem ser, por exemplo, a facilidade ou fluência com que um item é processado, a familiaridade com o item ou a pista enquanto estímulo (pista-alvo), a acessibilidade a informações parciais pertinentes à memória do item, a facilidade com que essa memória vem à mente, entre outras (Koriat, 2007). A tarefa construída neste estudo procura avaliar tanto JOLs quanto julgamentos de FOK, de modo que em estudos futuros essa hipótese possa ser testada em diferentes contextos e condições, conforme interesse do pesquisador ou avaliador.

Quanto às estratégias utilizadas para pesquisar o controle ou a regulação da memória, destaca-se a alocação de tempo de estudo (study time allocation ou STA), medida amplamente utilizada em metacognição 
e metamemória para avaliar controle (Ariel, Dunlosky \& Bailey, 2009; Dunlosky \& Connor, 1997; Metcalfe \& Kornell, 2003; Nelson \& Leonesio, 1988). Refere-se ao tempo que um indivíduo investe no estudo de um item até o ponto em que julga que conseguirá recuperá-lo posteriormente (Metcalfe \& Kornell, 2003). Alguns modelos teóricos já foram propostos, a fim de compreender os fatores que levam a STA. Atualmente, a hipótese da regulação com base na agenda (Ariel e cols., 2009) vem sendo debatida e sustenta que as metas estabelecidas pelo participante relativas à tarefa de memória é o fator principal que orienta o tempo de estudo. Dependendo das restrições e demandas características de cada tarefa (incluindo as fases de estudo e teste), o participante irá alocar maior ou menor tempo de estudo para cada item. Novamente, a tarefa elaborada aqui tem como objetivo avaliar esse processo de controle de memória, permitindo futuramente, conforme objetivos da avaliação, testar a hipótese da regulação com base na agenda para diferentes contextos.

Como monitoramento e controle são processos que ocorrem simultaneamente com os processos de memória, é importante que a avaliação nesse âmbito seja feita de forma online, como é feita na maioria das tarefas experimentais de metamemória. Nessas tarefas, os JOLs e julgamentos de FOK são coletados através das respostas a uma escala de 0 a 100 pontos (Eakin \& Hertzog 2012), ou a uma escala likert com pontos indicando o grau de "certeza" que a pessoa tem $(0 \%, 20 \%$, etc.; como em Krause \& Kennedy [2009]). A alocação de tempo de estudo (STA) pode ser determinada pelo tempo decorrido entre o início e o momento que o indivíduo escolhe finalizar o estudo do item.

A investigação em metamemória pode ser realizada pela análise das respostas do indivíduo para cada item apresentado ou por medidas mais globais e informativas acerca dos processos metamnemônicos. Essas medidas podem ser baseadas na precisão (precisão relativa e absoluta; Higham, 2002; Nelson \& Narens, 1990) e baseadas na sensibilidade (diferenças esperadas conforme características prévias dos itens; Matvey, Dunlosky \& Schwartz, [2006]). Para mais detalhes sobre outras medidas, ver Schraw (2008).

Em geral, o avaliador está interessado em saber se os processos de monitoramento estão sendo precisos quanto a informar o que está acontecendo no nível-objeto (por ex., desempenho de memória). Assim, podem ser calculadas a precisão relativa (relative accuracy) ou resolução (resolution) e a precisão absoluta (absolute accuracy) ou calibração (calibration). A primeira refere-se "ao grau em que o julgamento metacognitivo [...] prediz a probabilidade de desempenho [de memória] correto de um item em relação a outro" (Dunlosky \& Metcalfe, 2009, p .49). Assim, mensura-se a habilidade do indivíduo em discriminar seu desempenho conforme cada item. A precisão absoluta refere-se ao "nível em que as taxas dos julgamentos correspondem ao nível real de desempenho [de memória]" (Dunlosky \& Metcalfe, 2009 , p. 49). Isto é, uma alta precisão absoluta mostraria que, se o indivíduo em geral indicou, mediante de julgamentos, poucas chances de lembrar os itens (por ex., 30\%), seu desempenho final deve corresponder a esta proporção $(30 \%)$. No caso da precisão relativa, o cálculo pode ser feito pela correlação gama de Goodman-Kruskal, historicamente utilizada para julgamentos de metamemória (Nelson, 1984). A correlação varia de 0 a 1 , de modo que quanto mais próximo a $|1|$, mais precisos são os julgamentos.

A avaliação do controle de memória pode ser um pouco mais complexa do que a precisão do monitoramento, em função das características da tarefa (conforme sugerem Ariel e cols., 2009). Contudo, em uma tarefa que não envolve restrição de tempo para o estudo dos itens (momento em que os processos de controle atuam), é possível o exame da STA por meio de correlações. Estas são testadas entre o tempo de estudo de cada item e os JOLs ou os escores de desempenho de memória para estes itens (Dunlosky \& Connor, 1997). Tanto o monitoramento quanto o controle podem ser investigados através de medidas baseadas na sensibilidade. Testes de comparação de médias (ou seus correlatos não paramétricos) têm sido empregados para verificar se os julgamentos (Moulin, Perfect, Akhtar, Williams \& Souchay, 2011) e/ou a STA (Metcalfe \& Kornell, 2003) são sensíveis, isto é, diferentes para itens a priori distintos. Essa diferença, a priori, é manipulada pelo pesquisador através de, por exemplo, pares de palavras com $v$ s. sem relação semântica entre si ou palavras aprendidas $v$ s. palavras não aprendidas (Son \& Metcalfe, 2000), entre outros. Para tanto, deve haver um relativo controle de outras características do item que possam interferir no desempenho da tarefa de modo interveniente para fins do cálculo da sensibilidade. Por exemplo, variáveis psicolinguísticas como frequência de uso na língua e concretude de estímulos verbais que influenciam no desempenho mnemônico (Nelson, McKinney, Gee \& Janczura, 1998) e, consequentemente, no balanço entre monitoramento e controle.

A tarefa experimental de metamemória que será apresentada no presente artigo foi construída com base em dois modelos experimentais da área, quais sejam: 
as tarefas de múltiplas tentativas de estudo-teste utilizadas por Dunlosky e Connor (1997) e o paradigma recall-judge-recognition (RJR), oriundo do estudo de Hart (1965) e, posteriormente, utilizado por outros autores (Koriat, 1993; Marquié \& Huet, 2000). O primeiro modelo aplica uma etapa de estudo inicial de alguns ou todos os itens envolvidos na tarefa, seguida (JOLs tardios) ou acompanhada (JOLs imediatos) de uma etapa de julgamentos metamnemônicos sobre os itens e, então, uma etapa de recuperação. Subsequentemente, há nova sequência de estudo, julgamento e recuperação, podendo variar o número de vezes que isso é repetido. Já no paradigma RJR, o participante realiza, em geral, a recuperação (livre ou com pista) de um material estudado e, para os itens não recuperados corretamente, o participante realiza julgamentos de FOK, seguidos de uma etapa de reconhecimento. Essa última etapa pode ser do tipo múltipla escolha, em que o examinando tenta reconhecer o alvo dentre distratores (Thomas e cols., 2012).

O principal motivo de terem sido utilizados esses dois modelos experimentais foi fornecer tanto medidas de monitoramento (JOLs e FOK) quanto de controle de memória intratarefa, para fins de comparação. Apesar de a literatura descrever, por exemplo, fatores que influenciam ambos os julgamentos, conforme referido por Koriat (1997), a natureza das tarefas que avaliam esses processos diferem fundamentalmente. JOLs podem contar com treinos de recuperação, mesmo que de modo não declarativo; isto é, itens que são lembrados podem ter sido também lembrados durante o julgamento (Rhodes \& Tauber, 2011). No caso dos julgamentos de FOK, em razão de serem realizados apenas para itens não recuperados corretamente, não podem contar com treino de recuperação. Além disso, o tipo de tarefa de memória base para cálculo da precisão dos julgamentos é diferente (recuperação para JOLs vs. reconhecimento para julgamentos de FOK).

Outra manipulação experimental que também sinaliza fatores distintos para o monitoramento é o tipo de JOL realizado. JOLs imediatos (realizados segundos após a codificação) são menos precisos, em geral, do que JOLs tardios (realizados minutos ou horas após a codificação). Rhodes e Tauber (2011), em uma meta-análise, concluem que é possível que uma interação e acesso à informação de memória de longo prazo maior nos JOLs tardios conduzem à maior precisão desses julgamentos. Assim, considerando-se a abordagem da utilização de pistas (Koriat \& Levy-Sadot, 2001), esses julgamentos acessariam pistas distintas em comparação aos JOLs imediatos, a fim de se tornarem mais preditivos do desempenho de memória. Em suma, com essas manipulações objetiva-se uma avaliação mais abrangente do monitoramento, bem como do controle.

Neste artigo foram realizados três estudos para o estabelecimento de uma tarefa que refletisse o modelo teórico de Nelson e Narens (1990) e os paradigmas experimentais utilizados por Dunlosky e Connor (1997) e Hart (1965), e que fosse possível de aplicação em diferentes situações. Por exemplo, com amostras não clínicas (Hertzog, Sinclair \& Dunlosky, 2010), a quantidade necessária de itens pode ser maior do que em amostras clínicas (Krause \& Kennedy, 2009), mesmo o estímulo sendo da mesma natureza. Isso em função de efeitos de chão ou teto decorrentes, por exemplo, das diferenças quanto à capacidade de armazenamento de memória de uma amostra e outra. O primeiro estudo trata da seleção de itens para compor a tarefa. Os demais estudos são estudos-piloto a fim de verificar a adequação da tarefa (quantidade de itens, instruções, forma de registro, etc.) e testar algumas hipóteses do funcionamento metamnemônico, como discriminabilidade dos itens (Moulin e cols., 2011), precisão relativa entre os julgamentos metacognitivos e os escores de memória (Dunlosky \& Metcalfe, 2009) e maior precisão de JOLs tardios (Rhodes \& Tauber, 2011).

Estudo 1: seleção dos itens para a tarefa de metamemória

Este primeiro estudo teve como objetivo selecionar itens para compor a tarefa experimental de metamemória, que sejam adequados para manipulações experimentais e com homogeneidade quanto a características psicolinguísticas. $\mathrm{O}$ objetivo último foi criar itens para avaliação de memória (dentro do experimento de metamemória) através de evocação com pista e também reconhecimento. Também se pretendeu selecionar distratores para o reconhecimento. Desse modo, as características e critérios de seleção serão descritos no Método, e nas seções Resultados e Discussão serão apresentados os resultados dessa seleção e a lista de itens da tarefa experimental de metamemória.

\section{Método}

Foram utilizados pares de palavras como estímulos para a tarefa (Salles e cols., 2008). Esse tipo de material é amplamente utilizado em experimentos de memória e metamemória (Dunlosky \& Connor, 1997; Eakin \& Hertzog, 2012). A fim de proporcionar medidas para monitoramento e controle, e buscando diminuir a influência de variáveis intervenientes, os 
itens selecionados tiveram como base as seguintes variáveis: relação semântica (categórica e temática) a partir de normas de associação semântica de palavras (Salles e cols., 2008), classe gramatical, extensão da palavra, concretude e frequência de ocorrência na língua escrita.

Houve necessidade do estabelecimento de prioridades quanto à pertinência de cada variável a ser controlada ou manipulada na tarefa experimental, em função da disponibilidade de palavras com normas de associação semântica. A Figura 2 apresenta essa ordem de prioridades (critérios) para a escolha dos itens. Com relação ao critério relação semântica, $50 \%$ dos pares deveriam possuir baixa força associativa, isto é, com força de associação pista-alvo menor ou igual a $10 \%$, conforme Coney (2002). Para esse critério, foram utilizados os bancos de associação de palavras de Salles e cols. (2008) e Stein e Gomes (2009). Os outros 50\% dos pares deveriam ser formados por uma pista e um alvo sem relações semânticas. Para isso, os pares foram criados e foram consultados três juízes cegos (duas estudantes de graduação em Psicologia e uma psicóloga formada, que não conheciam os objetivos reais da tarefa), que opinaram, utilizando uma escala likert de sete pontos, sobre o quanto os pares pista-alvo refletiam relações semânticas. Caso um dos juízes percebesse uma alta relação semântica (três pontos ou mais na escala), a pista seria substituída por outra palavra. Essa proporção de $50 \%$ e manipulação foram necessárias para investigar a sensibilidade dos processos de monitoramento e controle, bem como para gerar variabilidade quanto à dificuldade (no desempenho de memória) dos itens. Além da relação semântica, buscou-se selecionar apenas substantivos (critério classe gramatical, confirmado por meio de consulta ao dicionário da língua portuguesa), com no máximo três sílabas (critério extensão da palavra).

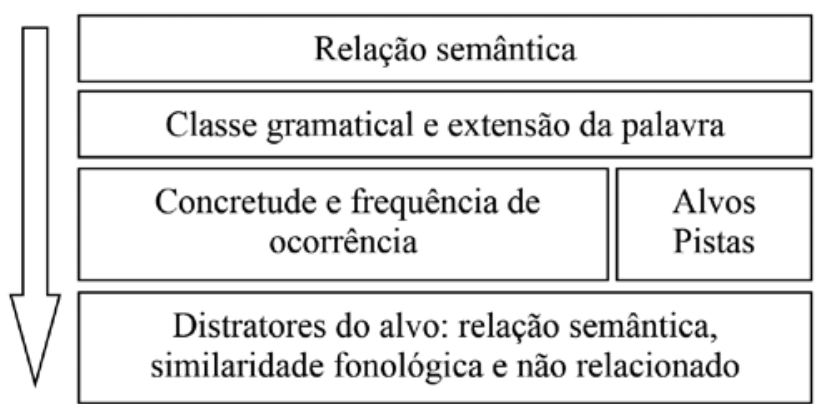

Figura 2. Critérios controlados na seleção dos itens da tarefa de metamemória.
Também se buscaram palavras com alto grau de concretude (critério concretude) e alta frequência na língua (critério frequência de ocorrência). Esse procedimento objetivou controlar a influência implícita que uma grande variabilidade de níveis de concretude e frequência dos itens teria nas tarefas de memória, o que poderia gerar confusão na interpretação dos resultados. Foi estabelecido um ponto de corte mínimo de 3,5 para concretude, tendo em vista que a escala original utilizada por Janczura, Castilho, Rocha, Van Erven e Huang (2007) variou de 1 a 7 . Para frequência, inicialmente foi estabelecido um ponto de corte mínimo de 100 ocorrências, conforme Kuhn, Abarca e Nunes (2000). Caso não fosse possível encontrar pares em que ambas as palavras (pista-alvo) se adequassem a esses critérios, foi dada prioridade ao alvo, tendo em vista que a pista é sempre apresentada nas etapas da tarefa e, portanto, seu processamento pode sofrer menor influência dessas variáveis psicolinguísticas.

Foram selecionados três tipos de distratores para cada alvo, para que se pudesse criar uma etapa de reconhecimento para avaliar os julgamentos de sensação de saber (FOK): relação semântica, similaridade fonológica e não relacionado ao alvo (sem relação semântica ou similaridade fonológica, porém apresentado junto a outra pista durante a tarefa). A relação semântica foi obtida a partir dos bancos de associação semântica de palavras de Salles e cols. (2008) e Stein e Gomes (2009), através da escolha de outro associado. Distratores fonológicos foram obtidos mediante palavras que rimam, têm mesma sílaba inicial ou alguma outra semelhança fonológica com o alvo. A fim de garantir a neutralidade (tanto semântica quanto de semelhança fonológica) dos distratores não relacionados, após os itens serem selecionados pelo experimentador, também foram consultados os mesmos juízes descritos anteriormente. Nesse momento, utilizando a mesma escala de sete pontos, os juízes opinavam sobre o quanto os pares alvo-distrator refletiam relações semânticas e/ou similaridade fonológica (quanto maior o valor da escala, maior semelhança possuíam). Caso a semelhança fosse percebida por um dos juízes (valor de três pontos ou mais), o alvo seria recombinado com outro distrator.

\section{Resultados}

Foram obtidos 100 pares de palavras (pista-alvo), apresentados no Anexo 1. Salienta-se que o número total de itens efetivamente incluídos na tarefa será apresentado nos Estudos 2 e 3, e depende de certas 
circunstâncias, conforme será discutido a seguir. Destes, 60 foram formados de palavras com até duas sílabas (exceto para o par "toalha-banho") e 40 pares de palavras de três sílabas (exceto os pares "braço-músculo", "frasco-veneno", "pelo-cachorro" e "branco-hospital"). Em apenas um item ("roubo-ladrão"), a força de associação entre pista e alvo foi maior do que $10 \%$ (valor de 27\%). Quanto à frequência de ocorrência, apenas o par "pata-grade" contou com um alvo cuja frequência é menor que 100 ocorrências (valor de 46, conforme normas de Kuhn e cols., [2000]). Ainda, essa característica psicolinguística teve índices bastante variados entre os estímulos alvo e pista. Quanto à concretude, para outros quatro pares ("água-filme", "trem-mãe", "rabo-álcool" e "vinho-mosca") não foram encontradas normas para os alvos. A análise de juízes resultou na recombinação de alguns pares alvo-distrator não relacionados, visto que pelo menos um dos juízes sinalizou haver proximidade semântica entre os pares. Não houve alterações quanto aos pares pista-alvo não relacionados, pois nenhum juiz indicou escore maior ou igual a três pontos na sua avaliação. A Tabela 1 apresenta dados descritivos dos 100 pares de palavras obtidos.

\section{Discussão}

Pode-se concluir que os itens selecionados para compor a tarefa experimental de metamemória apresentaram balanço adequado quanto à relação semântica entre pista e alvo, muito semelhante a outros estudos da literatura (Schmitter-Edgecombe \& Anderson, 2007). Isso permite a manipulação quanto ao tipo de item e a posterior medida de discriminação (Moulin e cols., 2011). Tanto pistas quanto alvos possuem, em média, altos índices de concretude, o que tende à homogeneidade, por exemplo, quanto ao uso de estratégias (neste caso, criar uma imagem mental da palavra). Apesar de para quatro pares não terem sido encontradas normas de concretude para os alvos, garantiu-se que todas as palavras fossem substantivos considerados concretos, evitando verbos, advérbios e outras classes gramaticais, em geral mais abstratas. A frequência de ocorrência alta para a maioria dos itens evita que o significado das palavras seja desconhecido para os participantes. Sobre a alta variabilidade dessa medida, destaca-se que o corpus de Kuhn e cols. (2000) baseia-se em frequência escrita, o que sinaliza que essa variabilidade pode não representar per se uma variabilidade na linguagem falada. Até o presente momento, não se conhece nenhum banco de normas de frequência de uso na linguagem falada para o português brasileiro.

Foram estabelecidos 100 pares de palavras, porém dependendo da população-alvo, a quantidade de pares de palavras pode variar. Estudos com adultos e idosos não clínicos utilizam um número maior de itens (ex., 60 pares em Hertzog e cols., 2010) do que estudos com pacientes clínicos, por ex., com síndrome disexecutiva (20 pares em Pinon, Allain, Kefi, Dubas \& Gall, 2005). A escolha dos itens pode ser guiada pelos critérios de seleção de itens apresentados na Figura 2, de modo que pares como "roubo-ladrão", que tiverem maior força de associação semântica que os demais pares, desfavorecendo a homogeneidade dos itens, possam ser evitados. No entanto, é necessário um estudo piloto para saber a quantidade adequada de itens, considerando-se as especificidades e complexidade da tarefa em questão, bem como diferentes populações-alvo.

Estudo 2: investigação do processo de monitoramento de memória

Neste momento buscou-se realizar um primeiro estudo piloto de modo a verificar a adequação dos pares de palavras selecionados, dos procedimentos de aplicação e do número necessário de pares a serem utilizados durante a tarefa experimental de metamemória. A seção Método detalha a amostra, a versão da tarefa utilizada como teste e as análises de dados, seguidas dos Resultados e Discussão.

\section{Método}

\section{Participantes}

Participaram do estudo, através de amostragem por conveniência, dez adultos (média de idade $=24,3$; $D P=4,2$ ), sendo sete estudantes de graduação em psicologia, um estudante de mestrado e um de doutorado em psicologia, e um recém-graduado em artes plásticas. Destes, cinco pertenciam ao sexo masculino.

\section{Procedimentos e materiais}

O projeto teve aprovação do Comitê de Ética em Psicologia da Universidade Federal do Rio Grande do Sul, protocolo número 21717. Os participantes responderam a tarefa, utilizando o software E-Prime 2 (Schneider, Eschman \& Zuccolotto, 2002a, 2002b), em sua primeira versão com 30 itens (50\% com e 50\% sem relação semântica). Foram usados apenas pares cujas palavras possuíam no máximo duas sílabas, provenientes dos 100 itens do Estudo 1. A versão da tarefa 
utilizada neste estudo piloto é apresentada no esquema da Figura 3. As perguntas em alguns retângulos estão resumidas neste esquema. A escala likert para os julgamentos foi apresentada indicando o significado de cada ponto. O tempo médio de aplicação da tarefa foi de 20 minutos por participante. Inicialmente, foi realizado um treino, em que foram apresentados três pares não incluídos posteriormente na tarefa, que consistiu nas fases de estudo, julgamentos de aprendizagem (JOL) tardios e recuperação com pista. Após o treino, independente do escore alcançado, a tarefa tinha início, com apresentação aleatória dos itens, tanto nas fases de estudo, quanto em julgamentos metacognitivos ou teste de memória.

Inicialmente, os pares (pista-alvo) foram apresentados para estudo, por cinco segundos cada. Posteriormente, o participante realizou JOLs tardios, com tempo livre, em que se deveria "julgar o quanto acha que conseguirá lembrar a segunda palavra do par daqui a pouco" (apenas a pista foi apresentada, conforme Figura 3). Uma escala likert de quatro pontos foi utilizada para os JOLs: $1=$ com certeza não vou lembrar; $2=$ é pouco provável que eu vá lembrar; $3=e ́$ bastante provável que eu vá lembrar; e $4=$ tenho certeza de que vou lembrar. $\mathrm{O}$ participante respondia utilizando os números 1, 2, 3 e 4 do teclado padrão do notebook.

Um teste de recuperação com pista, como medida de memória, foi realizado, e o participante deveria "tentar dizer em voz alta qual era a segunda palavra de cada par" (apenas a pista foi apresentada), sendo instruído a adivinhar quando não tivesse certeza. As respostas foram registradas pelo pesquisador em um teclado numérico a parte $(1=$ correta; $0=$ incorreta), para evitar feedback, além de as respostas incorretas serem anotadas em protocolo físico para futura análise qualitativa. Para os pares não recuperados corretamente, foram feitos julgamentos de sensação de saber (FOK) sobre "o quanto acha que conseguirá reconhecer a resposta certa para cada par". Para isso, foi esclarecido que "serão apresentadas a pista e quatro alternativas de resposta, sendo uma correta e as outras três distratoras". Portanto, o examinando realizou, para cada par, o

Tabela 1. Dados descritivos de 100 itens selecionados para a tarefa experimental de metamemória

\begin{tabular}{llccccc}
\hline & & $n$ & Média & $D P$ & Mínimo & Máximo \\
\hline Pares sem relação & Concretude pistas & 32 & 6,28 & 0,60 & 4,11 & 6,88 \\
semântica & Concretude alvos & 46 & 5,82 & 0,94 & 3,52 & 6,89 \\
& FO pistas & 50 & 1587,6 & 3175,9 & 84,0 & 18176,0 \\
& FO alvos & 50 & 3695,6 & 5719,9 & 46,0 & 25120,0 \\
Pares com relação & Concretude pistas & 30 & 5,80 & 0,91 & 3,63 & 6,86 \\
semântica & Concretude alvos & 50 & 5,90 & 0,92 & 3,62 & 6,93 \\
& FO pistas & 50 & 3194,0 & 6488,1 & 63,0 & 42152,0 \\
& FO alvos & 50 & 2024,2 & 2454,3 & 167,0 & 11975,0 \\
\hline
\end{tabular}

Nota: FO: frequência de ocorrência escrita na língua.

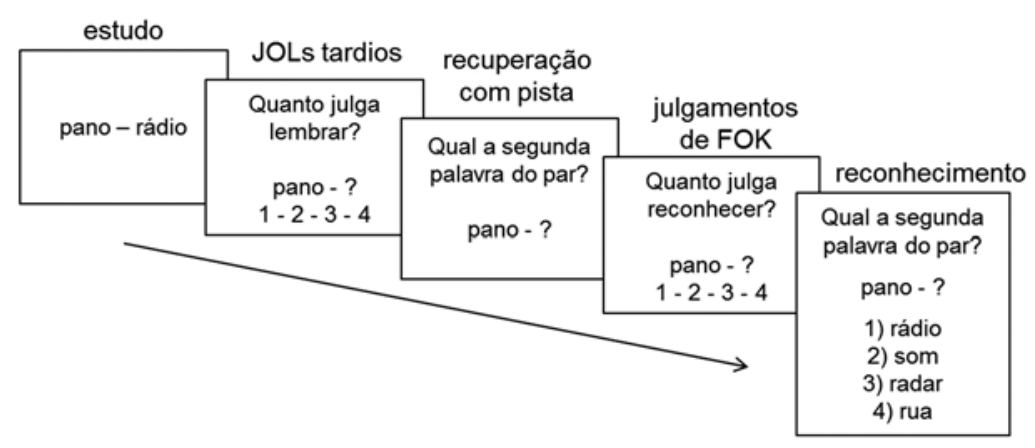

Figura 3. Paradigma experimental da tarefa de metamemória utilizado no primeiro estudo piloto 
julgamento seguido da tentativa de reconhecimento do alvo dentre quatro alternativas (teste de escolha forçada de múltiplas opções de resposta). Os distratores foram aleatoriamente distribuídos para cada pista previamente e possuíam ou relação semântica, ou fonológica ou nenhuma relação com o alvo. Todos os estímulos foram apresentados horizontalmente na tela do computador, centralizados, em cor preta e fundo branco, em fonte Arial 26. As demais informações (como instruções) foram apresentadas em fonte tamanho 22.

\section{Análise de dados}

Foram realizadas análises descritivas para as magnitudes dos julgamentos de FOK e JOLs tardios e os escores de memória (recuperação com pista e reconhecimento). Também se empregaram correlações gama de Goodman-Kruskal (Nelson, 1984) para o cálculo da precisão relativa dos julgamentos de FOK e JOLs tardios e teste de comparação de postos para amostras repetidas Wilcoxon, para investigar a sensibilidade dos julgamentos (pares com vs. sem relação semântica).

\section{Resultados}

A Tabela 2 apresenta dados (média e desvio padrão) de monitoramento e desempenho de memória do grupo na tarefa de metamemória. Conforme a Tabela 3, pôde-se observar a variabilidade da magnitude dos índices gama entre os participantes. Para os julgamentos de FOK, em muitos casos houve poucos itens a serem julgados e reconhecidos (só são reapresentados os itens não recordados na recuperação com pista), em função de escores altos na recuperação com pista (para os casos 2 e 8 o desempenho foi de 100\%). Desse modo, os dados para julgamento de FOK e reconhecimento foram suprimidos deste estudo. Já para os JOLs tardios, o número de itens foi adequado. O teste de Wilcoxon mostrou que pares com relação semântica receberam significativamente JOLs tardios mais baixos do que pares sem essa relação $[W(9)=-2,67 ; p=0,008$; tamanho de efeito $r=0,8]$.

\section{Discussão}

Conforme a Tabela 2, o valor médio dos JOLs foi de 0,28 , o que contrasta com a literatura, que aponta que JOLs tardios possuem alta precisão (em torno de gamma $=0,93$, conforme Rhodes e Tauber [2011]). Levanta-se a hipótese de que por ter sido uma aplicação inicial, as instruções não deixaram claro quando e como os pares deveriam ser recuperados. Além disso, o número amostral de dez pessoas é naturalmente pouco representativo da população de adultos jovens saudáveis. Mesmo assim, observou-se efeito de sensibilidade do tipo de item (par com e sem relação semântica). Ou seja, os participantes julgaram que pares mais fáceis de serem lembrados seriam efetivamente lembrados, o que indica que a manipulação da relação semântica entre os pares parece estar de acordo com a literatura (Moulin e cols., 2011).

A partir deste estudo observou-se a necessidade de algumas modificações na tarefa experimental. Primeiro, aumentar o número de itens, para que não ocorresse efeito de teto na recuperação com pista, como foi o caso aqui. Do contrário, a fase de reconhecimento, onde apenas itens não recuperados corretamente são incluídos, sofrerá impacto negativo. Acredita-se que com um aumento do número de itens, também seja possível incluir uma segunda fase de estudo (reestudo), a fim de obter uma medida de controle de memória, como

Tabela 2. Análise descritiva das medidas obtidas durante a tarefa de metamemória com universitários ( $\mathrm{n}=10$ )

\begin{tabular}{lccc}
\hline & $\mathrm{M}(D P)$ & Mínimo & Máximo \\
\hline Precisão relativa dos JOLs tardios & $0,28(0,13)$ & 0,04 & 0,50 \\
$\%$ recuperação com pista & $43,7(24,7)$ & 16,7 & 83,3 \\
\hline
\end{tabular}

Tabela 3. Correlações gamma da precisão relativa dos JOLS tardios e dos julgamentos de FOK para cada participante

\begin{tabular}{lcccccccccc}
\hline Participante & 1 & 2 & 3 & 4 & 5 & 6 & 7 & 8 & 9 & 10 \\
\hline Precisão relativa dos JOLs & $-0,50$ & 0,04 & 0,26 & 0,32 & 0,23 & $-0,42$ & 0,30 & 0,14 & $-0,18$ & 0,37 \\
Precisão relativa dos FOK & 0,83 & - & 1,00 & 0,84 & 1,00 & 0,20 & 0,86 & - & $-0,33$ & 0,50 \\
\hline
\end{tabular}


alocação de tempo de estudo (STA). Portanto, um novo estudo piloto foi necessário. Ademais, revisões de algumas instruções também foram necessárias, para que a tarefa se tornasse de fácil compreensão. Por exemplo, foi adicionado um exemplo de um par fictício (arroz-feijão), a fim de esclarecer, oralmente, que nas etapas de recuperação, apenas a primeira palavra seria apresentada, e o participante deveria lembrar qual era a segunda. Alguns elementos da tarefa, contudo, foram mantidos por apresentarem características satisfatórias, como o tipo de escala likert utilizada para os julgamentos e a variação no tipo de item (com e sem relação semântica).

Estudo 3: investigação dos processos de monitoramento e controle de memória

Este segundo estudo piloto teve como objetivo verificar a adequação da tarefa aumentando-se o número de itens e incluindo-se uma etapa de reestudo (para mensuração do tempo de estudo ou STA) e uma nova etapa de recuperação com pista. Ou seja, os paradigmas experimentais de múltiplas tentativas de estudo-teste (Dunlosky \& Connor, 1997) e de recall-judge-recognition (RJR; Hart, 1965) foram empreendidos conjuntamente aqui. Também se objetivou testar dois tipos de JOLs (tardios e imediatos) e verificar a adequação da tarefa para dois idosos e um paciente que sofreu Acidente Vascular Cerebral (AVC).

\section{Método}

\section{Participantes}

Participaram deste estudo piloto, mediante amostragem por conveniência, doze universitários (idade média $=19,5 ; D P=2,3)$ estudantes de graduação em psicologia. Além destes, participaram dois idosos neurologicamente saudáveis (idoso 1: mulher, 66 anos de idade, 4 anos de estudo; idoso 2: mulher, 72 anos, 5 anos de estudo) e um adulto que sofreu AVC e possui lesão no hemisfério cerebral esquerdo, já em fase crônica (homem, 79 anos, 8 anos de estudo), todos provenientes da população em geral.

\section{Procedimentos}

Versões diferentes da tarefa foram aplicadas, conforme será descrito abaixo, a fim de observar como os participantes respondiam e se havia variância nos dados. Os universitários responderam à tarefa experimental de metamemória com 50 itens, no formato conforme a Figura 4. Foram incluídas novas fases, como o reestudo, para mensuração da STA. Semelhante ao Estudo 2, o participante realizou um treino inicial, e foi submetido à fase de estudo dos 50 pares, julgamento de aprendizagem (JOL) e recuperação com pista. Em seguida, o participante realizou um reestudo (pista e alvo apresentados) autoguiado, no qual foi instruído a "pressionar um botão assim que julgar que já estudou o par de palavras o suficiente para poder lembrar a segunda palavra quando somente a primeira for apresentada daqui a pouco". Portanto, posteriormente o examinando realizou nova tentativa de recuperação com pista. Para os pares não recuperados nesta fase (recuperação com pista), o indivíduo realizou julgamentos de sensação de saber (FOK) e tentou o reconhecimento do alvo, conforme já apresentado no Estudo 2.

Além disso, duas condições de JOLs foram estabelecidas, sendo esta uma manipulação entre-participantes. Seis pessoas realizaram JOLs tardios, conforme já descrito no Estudo 2. Outras seis

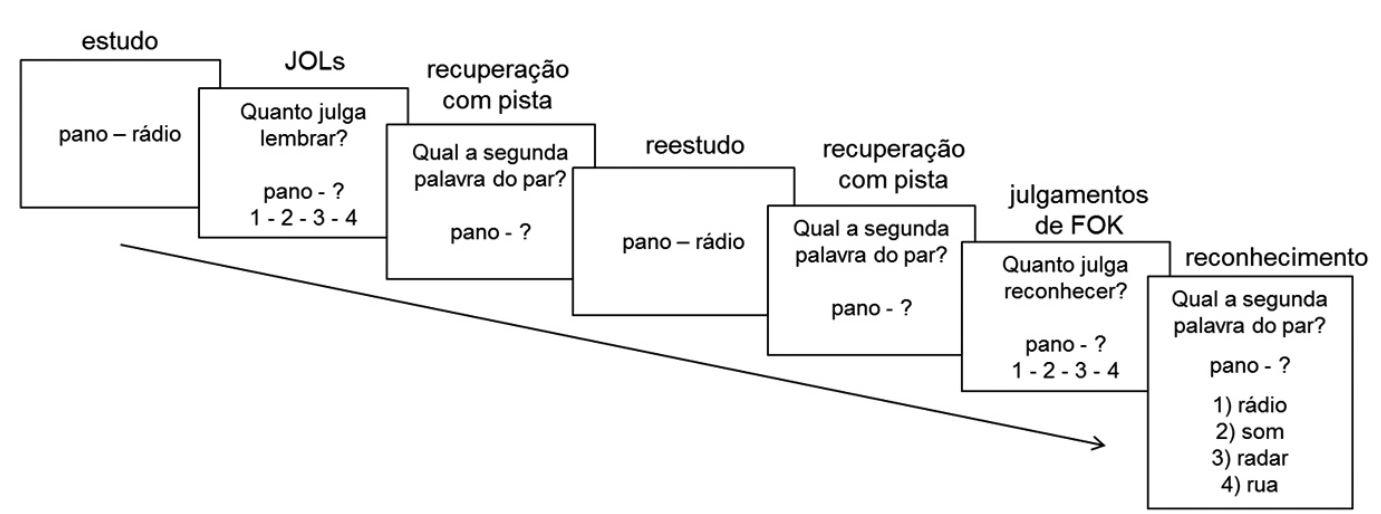

Figura 4. Esquema das etapas da tarefa experimental de metamemória no Estudo 3 e em sua versão final. As escalas likert (fases de JOLs e julgamentos de FOK) foram apresentadas nas cores vermelho (número 1), laranja (2), verde (3) e azul (4) e com o significado de cada ponto, para facilitar a compreensão. 
realizaram JOLs imediatos, de modo que após cada par ser estudado pela primeira vez, o participante realizou o julgamento do mesmo. No caso clínico, a mesma versão dos universitários com JOLs tardios foi utilizada, porém não foram realizados os julgamentos de FOK e o reconhecimento, o que será discutido a seguir. Para o Idoso 1, uma versão com 30 itens foi aplicada, mantendo-se as características de 50\% dos pares com relação semântica e todos os pares sendo reapresentados no reestudo. Para o Idoso 2, uma versão com 20 itens foi aplicada, e apenas 10 destes itens (cinco com e cinco sem relação semântica), selecionados previamente, foram reapresentados no reestudo, a fim de evitar efeito de teto na segunda recuperação com pista (após o reestudo). Todos os julgamentos foram feitos oralmente, e o avaliador registrou a resposta no software.

\section{Análise de dados}

Foram aplicados os mesmos tratamentos estatísticos descritos no Estudo 2, exceto que, na comparação entre julgamentos tardios e imediatos, o teste de comparação de postos para amostras independentes Mann-Whitney foi utilizado, tendo em vista que essa manipulação foi entre-participantes.

\section{Resultados}

Na Tabela 4 encontram-se os dados descritivos das medidas de monitoramento e controle de memória e o desempenho de memória na amostra dos universitários. A Tabela 5 apresenta os dados comparando-se as condições JOLs imediatos e tardios. O teste Mann-Whitney mostrou que os JOLs tardios foram estatisticamente mais precisos, em geral, que os JOLs imediatos. Ainda, a magnitude média dos JOLs tardios foi mais elevada que dos imediatos, porém apenas no caso dos pares sem relação semântica.

Com relação aos julgamentos de FOK dos universitários, novamente, houve efeito de teto na recuperação com pista, neste caso, após o reestudo, o que levou a poucos pares para cada participante julgar (através de FOKs) e reconhecer (pelo menos cinco participantes julgaram e reconheceram menos de três pares). Portanto, a análise estatística desses dados foi suprimida neste estudo. Essa situação foi considerada na versão final da tarefa, apresentada a seguir. No caso da STA, mensurada através do reestudo, não houve efeito de sensibilidade para todos os participantes. Em apenas sete, dos doze universitários, a média do tempo de reestudo foi maior para pares sem relação do que

Tabela 4. Análise descritiva das medidas obtidas na tarefa de metamemória com universitários $(\mathrm{n}=12)$

\begin{tabular}{lccc}
\hline & $\mathrm{M}(\mathrm{DP})$ & Mínimo & Máximo \\
\hline Precisão relativa (gamma) dos JOLs & $0,77(0,27)$ & 0,11 & 1,00 \\
\% recuperação com pista I & $55,2(14,6)$ & 28,0 & 72,0 \\
STA pares sem relação semântica & $5147,5(2540,3)$ & 2249,5 & 11053,7 \\
STA pares com relação semântica & $3236,87(2325,2)$ & 1251,1 & 10060,6 \\
\% recuperação com pista II & $87,7(12,4)$ & 60 & 100 \\
\hline
\end{tabular}

Nota: recuperação com pista I=realizada antes do reestudo; recuperação com pista II: realizada após o reestudo.

Tabela 5. Comparações entre as condições JOLS imediatos e tardios da tarefa de metamemória entre participantes universitários

\begin{tabular}{|c|c|c|c|c|c|}
\hline & JOLs imed & JOLs tardios & \multirow[t]{2}{*}{ Mann-Whitney } & \multirow[t]{2}{*}{$p$} & \multirow{2}{*}{$\begin{array}{l}\text { Tamanho } \\
\text { do efeito }\end{array}$} \\
\hline & \multicolumn{2}{|c|}{$\mathrm{M}(D P)$} & & & \\
\hline Precisão relativa (gamma) dos JOLs & $0,72(0,19)$ & $0,96(0,38)^{\mathrm{a}}$ & $U=22,0 ; z=-2,5$ & 0,009 & 0,7 \\
\hline Magnitude dos JOLs pares não relacionados & $1,90(0,37)$ & $2,65(0,46)$ & $U=3,5 ; z=-2,3$ & 0,015 & 0,7 \\
\hline Magnitude dos JOLs pares relacionados & $3,57(0,23)$ & $3,13(0,42)$ & $U=7,5 ; z=-1,7$ & 0,093 & 0,5 \\
\hline Correlações entre JOL e STA & $-0,45(0,08)^{\mathrm{b}}$ & $-0,58(0,06)$ & $U=1,0 ; z=-2,3$ & 0,019 & 0,7 \\
\hline
\end{tabular}

Nota. ${ }^{\text {a,b: }}$ Estes grupos possuíam $n=5$ e $n=4$, respectivamente. O restante possuía 6 participantes. 
pares com relação semântica (Mann-Whitney; $p<0,05)$. Ainda, utilizando-se a correlação de Pearson, a STA correlacionou-se significativamente $(p<0,05)$ com os JOLs para dez dos doze universitários (média da magnitude da correlação $\left.r_{\text {medio }}=-0,5 ; D P=0,1\right)$ e em nove casos também se correlacionou com o escore da recuperação com pista anterior (média da magnitude da correlação $\left.r_{\text {médio }}=-0,6 ; D P=0,1\right)$.

A Tabela 6 apresenta dados do caso clínico pós-AVC e dos idosos 1 e 2. Para o caso clínico, o tempo de estudo ou STA não foi diferente, em postos médios, para pares com ou sem relação. Igualmente, não houve correlação entre os JOLs e os tempos de reestudo de cada par. Além disso, o tempo de aplicação da tarefa foi muito longo, de modo que não foram realizados os julgamentos de FOK e o reconhecimento.

No caso idoso 1, aplicação do paradigma com 30 itens, a análise da precisão relativa tanto dos JOLs quanto dos julgamentos de FOK não foram empreendidas em função dos dados. O escore foi zerado na primeira recuperação com pista e os julgamentos de FOK foram todos de valor 1. Não houve diferenças entre pares com e sem relação semântica para a STA, nem correlações entre STA e os JOLs ou STA e a primeira recuperação com pista. $\mathrm{Na}$ aplicação com 20 itens no idoso 2, houve relativa melhor adequação (sem efeito de teto ou piso) nas etapas de desempenho de memória. Acredita-se que a redução do número de itens para o Estudo 2 também auxiliou nesse sentido. $\mathrm{O}$ teste Mann-Whitney não mostrou diferenças de desempenho entre os pares com e sem relação semântica para a STA. Por fim, houve correlação entre STA e JOL de gama $=0,67$.

\section{Discussão}

Talvez a maior dificuldade deste estudo, bem como dos anteriores, tenha sido encontrar um número de pares de palavras ideal para uma ampla margem de diferenças individuais. Conforme apresentado na revisão de literatura, parece haver uma calibração do número de itens das tarefas experimentais conforme a população (Hertzog e cols., 2010; Krause \& Kennedy, 2009; Pinon e cols., 2005). No caso dos universitários, 50 pares foi um número insuficiente, o que comprometeu a análise dos julgamentos de FOK. Já para os idosos, 30 pares pode ser um número muito grande, tendo em vista as diversas fases da tarefa e as mudanças cognitivas com o envelhecimento (Salthouse, 1996). Ainda, no adulto com lesão pós-AVC, a tarefa, que contou com 50 itens na fase de estudo, foi longa e extenuante, conforme relato do próprio participante, o que pode explicar o baixo desempenho de memória e a falta de indícios de habilidades de monitoramento e controle. Outro agravante é que quanto menor o número de itens, menor a variabilidade entre-participantes, o que comprometeria a análise dos dados e, consequentemente, as medidas de precisão e sensibilidade. Obviamente, em uma análise de grupo, essas dificuldades podem ser amenizadas, porém num delineamento de caso, como o do idoso 1 , os resultados são inconclusivos (no que se refere à recuperação com pista, de escore 0 ).

Apesar disso, os resultados indicaram que alguns efeitos previstos na literatura ocorreram mesmo em uma amostra piloto, com poucos casos. É o caso das diferenças na precisão relativa entre JOLs tardios e imediatos (Rhodes \& Tauber, 2011). Além disso, a Tabela 5 indica que a média (para os cinco dos seis casos cujo gama foi significativo ao nível de 0,05$)$ da precisão relativa dos JOLs tardios teve valor esperado (Rhodes \& Tauber, 2011). Porém, a média dos JOLs imediatos foi superior ao esperado. Estes julgamentos são, em geral, menos precisos, com gamas entre $=0,30$ e 0,45 (Leonesio \& Nelson, 1990; Rhodes \& Tauber, 2011). Apenas para dois participantes a precisão aproximou-se ao esperado $(0,48$ e 0,52). Já quanto à magnitude desses

Tabela 6. Análises descritivas das medidas obtidas na tarefa de metamemória para o caso clínico e os casos idoso 1 e 2

\begin{tabular}{lccccc}
\hline & Recup. I & Recup. II & Recon. & $\begin{array}{c}\text { Precisão relativa } \\
\text { dos JOL }\end{array}$ & $\begin{array}{c}\text { Precisão relativa } \\
\text { dos FOK }\end{array}$ \\
\hline Caso clínico & $2 \%$ & $6 \%$ & $24 \%$ & n.s & - \\
Idoso 1 & $0 \%$ & $3 \%$ & $33 \%$ & - & - \\
Idoso 2 & $4 \%$ & $65 \%$ & $37 \%$ & 1,0 & n.s \\
\hline
\end{tabular}

Nota. Recup. I, II=recuperação com pista antes (I) e depois (II) do reestudo; Recon.=reconhecimento; n.s.=não significativo. Valores não apresentados indicam que a análise não foi possível, em função da distribuição dos dados. 
julgamentos, o efeito dos JOLs tardios em aumentar a confiança em lembrar os itens posteriormente, foi detectado apenas para pares sem relação semântica. Isso pode indicar que apenas estímulos mais difíceis foram favorecidos por julgamentos tardios.

Para o idoso 2, a associação entre STA e JOL de gama $=0,67$ indica que, em certo nível, o tempo de reestudo foi baseado nos JOLs. Já para os universitários, em média, o STA se correlacionou mais com a recuperação com pista do que com os JOLs. Conforme Dunlosky e Connor (1997), em oposição aos achados aqui apresentados, idosos contam mais com seu desempenho de memória no item do que com os JOLs para guiar o tempo de estudo. Porém, como não foi significativa a associação entre STA e a recuperação com pista no caso do idoso 2 , não se pode indagar se os JOLs ou o desempenho de memória dos itens foi mais importante neste caso específico. No caso dos universitários, é plausível supor que a maioria dos participantes regulou seu reestudo com base, em algum grau, tanto no monitoramento quanto no desempenho de memória prévio.

Por fim, duas versões da tarefa experimental de metamemória poderiam ser sugeridas, ambas adotando o esquema da Figura 4 apresentado anteriormente. A diferença principal entre as versões I e II (itens para cada uma detalhados no Anexo 1) consiste no número de itens ( 80 e 20 itens, respectivamente) e no tempo de exposição dos pares no Estudo 1 (cinco e oito segundos, respectivamente). Acredita-se que a versão I (80 itens) possa ser utilizada em adultos jovens (até 40 anos) e de meia idade (de 40 a 59 anos), com nível de escolaridade médio a alto. A versão II (20 itens) talvez seja propícia para adultos de meia idade de baixa escolaridade, idosos ou pessoas com comprometimento significativo das funções de atenção e memória. Em todas as etapas, os itens são apresentados aleatoriamente e o examinador deve fornecer oralmente instruções. A tarefa pode ser feita no software E-prime 2 (Schneider e cols., 2002a, 2002b) e aplicada individualmente. O tempo de aplicação pode ser bastante variado, entre 30 e 80 minutos, dependendo da velocidade do examinando responder, bem como da versão utilizada. Além disso, diversas etapas possuem tempo livre e as duas últimas etapas (julgamentos de FOK e reconhecimento) possuem um número variado de itens para cada participante, conforme o escore na recuperação com pista anterior. Estudos futuros poderiam obter um cenário mais realístico para aplicação da versão mais adequada, dependendo de outras circunstâncias não pesquisadas aqui (crianças, outros quadros neuropsicológicos ou psiquiátricos, etc.).

\section{Considerações finais}

Os resultados alcançados nos Estudos 1, 2 e 3 desta pesquisa sugerem a viabilidade da utilização de uma tarefa para avaliação online dos processos metamnemônicos de monitoramento e controle de memória mediante recuperação com pista e reconhecimento de pares de palavras com e sem relação semântica. Entende-se que os itens, os procedimentos e os resultados encontrados aqui sejam úteis para o exame mais pormenorizado das habilidades metacognitivas e metamnemônicas. É importante que, a partir desta pesquisa, os pesquisadores e profissionais que trabalham com avaliação cognitiva/neuropsicológica atentem para o papel dos julgamentos metamnemônicos e das estratégias e processos de controle no momento da avaliação da codificação e recuperação de informações. Tarefas como a utilizada aqui são presentes em outras áreas, como consciência fonológica, que já possui protocolos experimentais bem estabelecidos e validados (Lance, Swanson \& Peterson, 1997). Caso haja interesse na construção de outras tarefas experimentais para avaliação online de habilidades metacognitivas, os resultados apresentados aqui podem servir como dados norteadores da pesquisa.

Uma das limitações encontradas ao longo da construção da tarefa foi o ajuste do número de pares de palavras para cada participante. Considera-se que possam ocorrer casos em que a análise estatística paramétrica seja inadequada. $\mathrm{O}$ avaliador ou pesquisador que utilizar o paradigma deve estar ciente dessa limitação e, se possível, verificar previamente a capacidade de atenção concentrada e de armazenamento de curto prazo do avaliado. Com isto, poderá ter uma ideia do número de itens a ser inserido na tarefa. É possível que versões intermediárias (por ex., com 40 itens) sejam necessárias. Considera-se que paralelamente ao uso de tarefas de avaliação online, pode-se complementar a análise dos processos metamnemônicos com a avaliação off-line, por meio de escalas de autorrelato, como o Questionário de Metamemória em Adultos (MIA; (Yassuda, Lasca \& Neri, 2005), entre outros instrumentos.

\section{Referências}

Ariel, R., Dunlosky, J., \& Bailey, H. (2009). Agenda-based regulation of study-time allocation: when agendas override item-based monitoring. Journal of Experimental Psychology: General, 138(3), 432-447. doi:10.1037/a0015928. 
Chua, E. F., Schacter, D. L., \& Sperling, R. A. (2009). Neural correlates of metamemory: a comparison of feeling-of-knowing and retrospective confidence judgments. Journal of Cognitive Neuroscience, 21(9), 1751-1765. doi:10.1162/jocn.2009.21123.

Coney, J. (2002). The effect of associative strength on priming in the cerebral hemispheres. Brain and Cognition, 50, 234-241.

Dunlosky, J., \& Nelson, T. O. (1994). Does the sensitivity of judgments of learning (JOLs) to the effects of various study activities depend on when the JOLs occur? Journal of Memory and Language, 33, 545-565.

Dunlosky, J., \& Connor, L. T. (1997). Age differences in the allocation of study time account for age differences in memory performance. Memory \& Cognition, 25, 691-700. doi:10.3758/bf03211311.

Dunlosky, J., \& Metcalfe, J. (2009). Metacognition. Washington, DC: Sage Publications.

Eakin, D. K., \& Hertzog, C. (2012). Age invariance in feeling of knowing during implicit interference effects. The Journals of Gerontology, Series B: Psychological Sciences and Social Sciences, 67(5), 555-562.

Flavell, J. (1979). Metacognition and Cognitive Monitoring: a new area of cognitive-developmental inquiry. American Psychologist, 34(10), 906-911.

Hart, J. T. (1965). Memory and the feeling-of-knowing experience. Journal of Educational Psychology, 56, 208-216. doi:10.1037/h0022263.

Hertzog, C., Sinclair, S. M., \& Dunlosky, J. (2010). Age differences in the monitoring of learning: cross-sectional evidence of spared resolution across the adult life span. Developmental Psychology, 46(4), 939-948. doi:10.1037/a0019812.

Higham, P. (2002). Strong cues are not necessarily weak: Thomson and Tulving (1970) and the encoding specificity principle revisited. Memory \& Cognition, 30(1), 67-80.

Janczura, G. A., Castilho, G. M., Rocha, N. O., Van Erven, T. J. C., \& Huang, T. P. (2007). Normas de concretude para 909 palavras da língua portuguesa. Psicologia: Teoria e Pesquisa, 23, 195-204.

Koriat, A. (1993). How do we know that we know? The accessibility model of the feeling of knowing. Psychological Review, 100, 609-639.
Koriat, A. (1997). Monitoring one's own knowledge during study: a cue-utilization approach to judgments of learning. Journal of Experimental Psychology: General, 126(4), 349-370.

Koriat, A. (2007). Metamemory and consciousness. Em P. D. Zelazo, M. Moscovitch, \& E. Thompson (Eds.), The Cambridge Handbook of Consciousness (pp. 289-326). Nova Iorque: Cambridge University Press.

Koriat, A., \& Levy-Sadot, R. (2001). The combined contributions of the cue-familiarity and accessibility heuristics to feelings of knowing. Journal of Experimental Psychology: Learning, Memory, and Cognition, 27(1), 34-53. doi:10.1037//0278-7393.27.1.34.

Koriat, A., Ma'ayan, H., \& Nussinson, R. (2006). The intricate relationships between monitoring and control in metacognition: lessons for the cause-and-effect relation between subjective experience and behavior. Journal of Experimental Psychology: General, 135(1), 36-69. doi:10.1037/0096-3445.135.1.36.

Krause, M., \& Kennedy, M. R. T. (2009). Metamemory adjustments over time in adults with and without traumatic brain injury. Brain Injury, 23(12), 965-972. doi:10.3109/02699050903373485.

Kuhn, D., Abarca, E., \& Nunes, M. G. (2000). Corpus Nilc de português escrito no Brasil. Série de relatórios do Núcleo Interinstitucional de Linguística Computacional da USP, UFSCar e UNESP. Disponível em http://www.nilc.icm.usp.br/nilc/ publications.htm\#TechnicalReports.

Lance, D. M., Swanson, L. A., \& Peterson, H. A. (1997). A validity study of an implicit phonological awareness paradigm. Journal of Speech, Language \& Hearing Research, 40(5), 1002-10.

Leonesio, R. J., \& Nelson, T. O. (1990). Do different metamemory judgments tap the same underlying aspects of memory? Journal of Experimental Psychology-Learning Memory and Cognition, 16, 464-470. doi:10.1037//0278-7393.16.3.464.

Marquié, J. C., \& Huet, N. (2000). Age differences in feeling-of-knowing and confidence judgments as a function of knowledge domain. Psychology and $A g-$ ing, 15, 451-461. doi:10.1037//0882-7974.15.3.451.

Matvey, G., Dunlosky, J., \& Schwartz, B. (2006). The effects of categorical relatedness on judgements 
of learning (JOLs). Memory, 14(2), 253-261. doi:10.1080/09658210500216844.

Metcalfe, J., \& Kornell, N. (2003). The Dynamics of Learning and Allocation of Study Time to a Region of Proximal Learning. Journal of Experimental Psychology: General, 132(4), 530-542.

Moulin, C. J. A., Perfect, T. J., Akhtar, S., Williams, H. L., \& Souchay, C. (2011). Judgements of learning and study- time allocation: an illustration from neuropsychology. Em P. A. Higham \& L. P. Leboe (Eds.), Constructions of Remembering and Metacognition: Essays in Honour of Bruce Whittlesea (pp. 157-183). New York: Palgrave Macmillan.

Nelson, D. L., McKinney, V. M., Gee, N. R., \& Janczura, G. A. (1998). Interpreting the influence of implicitly activated memories on recall and recognition. Psychological Review, 105(2), 299-324.

Nelson, T. O. (1984). A comparison of current measures of the accuracy of feeling-of-knowing predictions. Psychological Bulletin, 95(1), 109-133.

Nelson, T. O., \& Leonesio, R. J. (1988). Allocation of self-paced study time and the "labor-in-vain effect." Journal of Experimental Psychology: Learning, Memory, and Cognition, 14(4), 676-686.

Nelson, T. O., \& Narens, L. (1990). Metamemory: A theoretical framework and new findings. The psychology of learning and motivation, 26, 125-173.

Pinon, K., Allain, P., Kefi, M. Z., Dubas, F., \& Gall, D. L. (2005). Monitoring processes and metamemory experience in patients with dysexecutive syndrome. Brain and Cognition, 57(2), 185-188. doi:10.1016/j. bandc.2004.08.042.

Rhodes, M. G., \& Tauber, S. K. (2011). The influence of delaying judgments of learning on metacognitive accuracy: a meta-analytic review. Psychological Bulletin, 137(1), 131-148. doi:10.1037/a0021705.

Salles, J. F., Holderbaum, C. S., Becker, N., Rodrigues, J. C., Liedtke, F. V., Zibetti, M. R., \& Piccoli, L. F. (2008). Normas de associação semântica para 88 palavras do português brasileiro. Revista PSICO, 39, 362-370.

Salthouse, T. A. (1996). The processing-speed theory of adult age differences in cognition. Psychological Review, 103(3), 403-428.
Schmitter-Edgecombe,M., \& Anderson,J.W. (2007).Feeling of knowing in episodic memory following moderate to severe closed-head injury. Neuropsychology, 21(2), 224-234. doi:10.1037/0894-4105.21.2.224

Schneider, W., Eschman, A., \& Zuccolotto, A. (2002a). E-Prime reference guide. Pittsburgh: Psychology Software Tools Inc.

Schneider, W., Eschman, A., \& Zuccolotto, A. (2002b). E-Prime user's guide. Pittsburgh: Psychology Software Tools Inc.

Schraw, G. (2008). A conceptual analysis of five measures of metacognitive monitoring. Metacognition and Learning, 4(1), 33-45. doi:10.1007/ s11409-008-9031-3.

Son, L. K., \& Metcalfe, J. (2000). Metacognitive and control strategies in study-time allocation. Journal of Experimental Psychology-Learning Memory and Cognition, 26, 204-221. doi:10.1037//0278-7393.26.1.204.

Stein, L. M., \& Gomes, C. F. de A. (2009). Normas brasileiras para listas de palavras associadas: associação semântica, concretude, frequência e emocionalidade. Psicologia: Teoria e Pesquisa, 25(4), 537-546. doi:10.1590/S0102-37722009000400009.

Thomas, A. K., Bulevich, J. B., \& Dubois, S. J. (2012). An analysis of the determinants of the feeling of knowing. Consciousness and Cognition, 21(4), 16811694. doi:10.1016/j.concog.2012.09.005.

Van Overschelde, J. P. (2008). Metacognition: knowing about knowing. Em J. Dunlosky \& R. A. Bjork (Eds.), Handbook of metamemory and memory (pp. 4771). Nova Iorque: Psychology Press.

Veenman, M. V. J., Van Hout-Wolters, B. H. A. M., \& Afflerbach, P. (2006). Metacognition and learning: conceptual and methodological considerations. Metacognition Learning, 1, 3-14.

Yassuda, M. S., Lasca, V. B., \& Neri, A. L. (2005). Meta-memória e auto-eficácia: um estudo de validação de instrumentos de pesquisa sobre memória e envelhecimento. Psicologia: Reflexão e Crítica, 18(1), 78-90. doi:10.1590/S0102-79722005000100011.

Recebido em: 19/04/2013

Reformulado em: 03/10/2013

Aprovado em: 24/02/2014 


\section{Anexo 1}

Lista de pares de palavras pista/alvo utilizados nas versões I e II da tarefa experimental de metamemória

\begin{tabular}{|c|c|c|c|c|c|c|c|c|c|c|c|}
\hline \multicolumn{5}{|c|}{ Pares sem relação semântica } & & \multicolumn{6}{|c|}{ Pares com relação semântica } \\
\hline \multirow{2}{*}{ Pista } & \multirow{2}{*}{ Alvo } & \multicolumn{3}{|c|}{ Distratores } & & \multirow{2}{*}{ Pista } & \multirow{2}{*}{ Alvo } & \multicolumn{3}{|c|}{ Distratores } & \\
\hline & & Assoc. & Fonol. & Neutro & & & & Assoc. & Fonol. & Neutro & \\
\hline lanche & câncer & tumor & canto & rua & $a$ & poste & rua & passeio & lua & câncer & $a$ \\
\hline cantor & nação & povo & mansão & prova & $a$ & selva & leão & caça & peão & rádio & $a$ \\
\hline pano & rádio & som & radar & tênis & $a, b$ & bola & tênis & meia & tela & nação & $a$ \\
\hline barba & dança & tango & lança & leão & $a, b$ & nota & prova & teste & cova & dança & $a, b$ \\
\hline botão & papel & lápis & anel & chá & $a$ & erva & chá & fumo & pá & papel & $a$ \\
\hline metal & rede & pesca & cede & bolo & $a$ & festa & bolo & torta & bala & rede & $a, b$ \\
\hline fruta & disco & fita & risco & guerra & $a$ & briga & guerra & luta & fera & disco & $a, b$ \\
\hline filme & água & poço & língua & mesa & $a, b$ & cor & azul & cinza & sul & beijo & $a$ \\
\hline lata & beijo & boca & queijo & azul & $a, b$ & peixe & mar & rio & ar & sangue & $a, b$ \\
\hline areia & sangue & veia & mangue & mar & $a$ & dedo & pé & mão & fé & carta & $a$ \\
\hline chapéu & carta & cartão & porta & pé & $a$ & sala & aula & quadro & jaula & pneu & $a, b$ \\
\hline cama & vidro & cristal & cedro & ponte & $a, b$ & janta & mesa & classe & presa & lixo & $a$ \\
\hline onça & flor & cravo & trator & água & $a, b$ & campo & serra & morro & ferro & gordo & $a$ \\
\hline mel & pó & chão & nó & bolso & $a, b$ & suco & gelo & neve & selo & grama & $a$ \\
\hline dente & pedra & rocha & pena & quarto & $a, b$ & cinto & calça & saia & valsa & calor & $a, b$ \\
\hline pulmão & leite & açúcar & leito & trono & $a$ & folha & livro & letra & livre & pata & $a, b$ \\
\hline prato & jardim & rosa & cetim & vaca & $a$ & carne & vaca & gado & faca & prisão & $a$ \\
\hline grade & pata & urso & mala & livro & $a$ & lona & circo & tenda & cerco & homem & $a$ \\
\hline muro & homem & moço & honra & circo & $a$ & carro & marca & tipo & arca & jardim & $a, b$ \\
\hline unha & prisão & cela & trovão & marca & $a$ & nuvem & chuva & sol & luva & farda & $a$ \\
\hline mãe & trem & vapor & trio & vinho & & roda & pneu & preto & liceu & vidro & $a, b$ \\
\hline colo & farda & traje & guarda & chuva & $a$ & & lixo & cesto & fixo & flor & $a$ \\
\hline vaso & moto & banco & foto & ponte & $b$ & banha & gordo & cheio & bordo & pó & $a$ \\
\hline álcool & rabo & ponta & cabo & motor & & suor & calor & quente & doutor & pedra & $a$ \\
\hline mosca & vinho & uva & ninho & moto & $b$ & capim & grama & verde & drama & leite & $a, b$ \\
\hline nariz & ponte & via & fonte & trem & & gato & bicho & verme & nicho & ladrão & $t$ \\
\hline teia & lotor & diesel & castor & rabo & $t$ & terra & fogo & chama & jogo & pão & $t$ \\
\hline porco & bolso & roupa & bolsa & serra & $a$ & roubo & ladrão & arma & padrão & banho & \\
\hline pai & quarto & casa & parto & calça & $a$ & & pão & massa & cão & & \\
\hline lama & trono & rei & sono & gelo & $a$ & toalha & banho & limpo & ganho & bicho & \\
\hline árvore & sorriso & risada & aviso & cadeira & $a$ & semente & laranja & $\operatorname{limão}$ & granja & animal & \\
\hline palhaço & castelo & torre & martelo & estado & & militar & soldado & quartel & tornado & borracha & $a$ \\
\hline tesouro & coração & vermelho & coleção & palácio & $a$ & vegetal & animal & boi & avental & músculo & \\
\hline presunto & estado & país & estátua & gigante & & braço & músculo & perna & crepúsculo & laranja & \\
\hline relógio & gordura & pastel & tortura & batalha & & indústria & máquina & trabalho & tática & agulha & $a$ \\
\hline criança & sapato & marrom & mulato & veneno & $a$ & sorvete & morango & doce & morador & cachorro & \\
\hline cigarro & banana & casca & cabana & inverno & $a$ & asfalto & estrada & viagem & escada & música & $a$ \\
\hline pimenta & barulho & trânsito & mergulho & panela & $a$ & estrela & planeta & cometa & caneta & menina & $a$ \\
\hline batata & agulha & injeção & agudo & cabelo & $a$ & & abraço & carinho & pedaço & cidade & $a$ \\
\hline calcanhar & borracha & desenho & bolacha & bandido & $a$ & escola & cadeira & assento & capoeira & comida & $a$ \\
\hline empresa & batalha & navio & navalha & castelo & & rainha & palácio & princesa & palanque & dentista & $a$ \\
\hline tesoura & cidade & comércio & metade & salada & $a$ & frasco & veneno & remédio & terreno & estudo & $a$ \\
\hline garrafa & menina & boneca & colina & aranha & $a$ & cobertor & inverno & casaco & inferno & dinheiro & $a$ \\
\hline carteira & música & violão & mosquito & hospital & $a$ & cozinha & panela & refeição & janela & revólver & $a$ \\
\hline antena & comida & almoço & corrida & sorriso & $a$ & escova & cabelo & cabeça & carneiro & soldado & $a$ \\
\hline carnaval & dentista & aparelho & artista & sapato & $a$ & polícia & bandido & cadeia & bandeira & máquina & $a$ \\
\hline cortina & estudo & colégio & estufa & coração & $a$ & tomate & salada & verdura & sacada & estrada & $a$ \\
\hline brinquedo & gigante & anão & diamante & gordura & & pelo & cachorro & Osso & cachoeira & morango & \\
\hline espelho & dinheiro & salário & pinheiro & banana & $a$ & picada & aranha & inseto & montanha & planeta & $a$ \\
\hline moeda & revólver & assalto & revolta & barulho & a & branco & hospital & saúde & mineral & abraço & $a$ \\
\hline
\end{tabular}

Nota. Assoc. =distrator associativo; Fonol. =distrator fonológico; Não rel.: distrator não relacionado ao alvo; $a=$ pares presentes na versão 1 (80 pares) da tarefa; $b=$ pares presentes na versão 2 (20 pares); $t=$ pares apresentados no treino. 
Sobre os autores:

Maxciel Zortea possui graduação em Psicologia, mestrado em Psicologia e atualmente é aluno de doutorado do Programa de Pós-Graduação em Psicologia da Universidade Federal do Rio Grande do Sul (UFRGS), com estágio de doutorado sanduíche pela University of Southampton, Southampton, Reino Unido. É membro do Núcleo de Estudos em Neuropsicologia Cognitiva (Neurocog) da UFRGS.

Graciela Inchausti de Jou possui graduação em Psicologia e mestrado e doutorado em Psicologia do Desenvolvimento pelo Programa de Pós-Graduação em Psicologia da UFRGS. Também possui pós-doutorado pelo mesmo programa. Atualmente é professora visitante vinculada ao Grupo de Pesquisa em Infância, Desenvolvimento e Psicopatologia (GIDEP) e ao grupo de pesqusia Neurocog da UFRGS.

Jerusa Fumagalli de Salles possui graduação em Fonoaudiologia, mestrado e doutorado em Psicologia do Desenvolvimento pela UFRGS. É professora adjunta no Instituto de Psicologia e do Programa de Pós-Graduação em Psicologia desta mesma universidade. Coordena o grupo de pesquisa Neurocog e o Curso de Especialização em Neuropsicologia da UFRGS, além de integrar a equipe do Ambulatório de Neuropsicologia do Hospital de Clínicas de Porto Alegre.

Contato com os autores:

Maxciel Zortea

Instituto de Psicologia da UFRGS

Rua Ramiro Barcelos, 2600, $1^{\circ}$ andar, sala 114, Bairro Santa Cecília

Porto Alegre, RS,

CEP: 90035-003.

E-mail:max.zortea@gmail.com

Telefone: 51 3308-5341 